SFB

Dual disadvantage and dispersion dynamics for

income distributions

823

Roger J. Bowden, Peter N. Posch,

Daniel Ullmann

Nr. 25/2016

$\omega$

$\mathcal{O}$

(

0

(1)

(D)

SFB
823
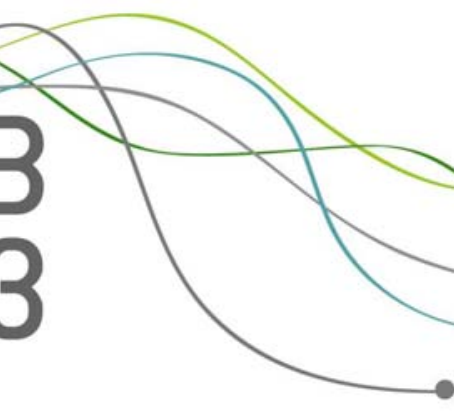



\title{
Dual disadvantage and dispersion dynamics for income distributions*
}

\author{
Roger J. Bowden ${ }^{\dagger}$, Peter N. Posch ${ }^{\ddagger}$, Daniel Ullmann ${ }^{\S}$
}

2. Juni 2016

\begin{abstract}
Income distribution has been a longstanding focus of social and economic interest, but never more so than in recent times. New metrics for disadvantage and spread enable a more precise differentiation of directional asymmetry and dispersion, drawing on an internal contextual perspective. The dual metrics for asymmetry and spread can be plotted over time into a phase plane, enabling comparative social welfare perspectives over time and between countries. The methods are utilised to study the dramatic changes that took place in Europe prior to and after the GFC. Major differences are revealed. In terms of asymmetry and spread, some countries have been fallers (lower in both) while other countries are risers.
\end{abstract}

Keywords: Asymmetry, economic disadvantage, income inequality, partition entropy, social welfare function

JEL Classification: D31, D63, I310, I320

\footnotetext{
${ }^{*}$ Disclaimer: The empirical analysis in this paper is based on data drawn from Eurostat, EU Statistics on Income and Living Conditions (2013). Thanks go to the Agency for its provision. However, the responsibility for all conclusions drawn from the data rests with the authors.

Acknowledgement: Financial support by the Collaborative Research Center: Statistical Modeling of Nonlinear Dynamic Processes (SFB 823) of the German Research Foundation (DFG) is gratefully acknowledged

${ }^{\dagger}$ Kiwicap Research ltd, Wellington, New Zealand, ph+64 44724094, fax 4983, Email: roger.bowden@kiwicap.co.nz, Corresponding author

¥Technical University of Dortmund, Faculty of Business, Economics and Social Science, 44227 Dortmund, Germany, E-Mail: peter.posch@udo.edu

$\S$ Technical University of Dortmund, Faculty of Business, Economics and Social Science, 44227 Dortmund, Germany, E-Mail: daniel.ullmann@udo.edu
} 


\section{Introduction}

Income inequality has been a perennial topic of economic and social interest, but never more so than the present, where dramatic changes have followed within just a short span of time. Performance driven management rewards seen as excessive have attracted much public attention. But other influences have been at least as pervasive. Technological displacement for middle management, import competition from cheaper emerging countries, free trade agreements, adverse fallouts from public spending bubbles, commodity price reversals, the global financial crisis, are all some of the causal influences, combining as the perfect storm in their fallouts for remuneration and employment down the line. The empirical content of the present paper, namely the ten years in Europe just prior to and succeeding the Global Financial Crisis (GFC), reflect changes of this sort. To what extent they represent welfare loss, and how to measure it, requires a methodology that is itself adapted to the economic and social context. Metrics are one thing; meaningful metrics in this context are quite another. The agenda of the present paper is to develop and apply such metrics to the experience of the dramatic, if not tumultuous, years between 2005 - 2013 in European countries.

In the most general sense, metrics for income distributions are part of a wider body of knowledge into social welfare functions developed and debated over many years. But establishing a consensus has not been easy. At the most abstract level, Goodman \& Arrow (1953) showed that the possibility of a consistent ordinal social utility as earlier envisaged by Bergson (1938) and Samuelson (1947), was limited at best and certainly would not extend to universal agreement among the subjects themselves as to a single best metric for income inequality. The empirics have therefore focused upon metrics for income inequality that appeal in designated ways to the observer's own preconceptions as to fairness. Still the best known and most widely accepted metric of this kind is the Gini coefficient, which measures the non-alignment of the accumulated percentage of income with the progressive numbers of the people enjoying it. As the Gini coefficient is not able to represent different social preferences Atkinson (1970), Donaldson \& Weymark (1980) and Yitzhaki (1983) propose a parametric extension, where the parameter can account for different preferences (Greselin \& Zitikis, 2015). Sen (1970) proposed a metric that combines in itself both the mean income and the complement (1-G) of the Gini index.

But the Gini has several downsides. One of the most obvious problems is the ambiguity of the coefficient itself. Since it reflects the integral of the difference of the $45^{\circ}$ line and the Lorenz curve, we can mirror the Lorenz curve on the orthogonal line that intersects the $45^{\circ}$ line in the middle, and end up with just the same Gini. Apart from this, there is a potential ambiguity problem arising in intertemporal or cross country comparisons. Davies \& Hoy (1995) point out that where two Lorenz curves intersect it may not be possible to rank the Gini coefficients, even using the same underlying social welfare function; only with further assumptions about the variance, this is possible. The problem 
is a general one and applies to all measures based on the Lorenz curves.

As a possible alternative, the Theil index (Theil, 1965) uses the distribution's Shannon entropy in order to measure the expected information content of the distribution, which is then interpreted as inequality. But as Sen (1970) puts it, "... the average of the logarithms of the reciprocals of income shares weighted by incomes shares [the Theil index] is not a measure that is exactly overflowing with intuitive sense"(Sen, 1970, p. 36). See also Conceição \& Ferreira (2000). On the other hand, the Theil index does obey the axiom of decomposability (Foster, 1983; Kraemer, 1998), which means that the measure for the whole distribution can be expressed as a weighted sum of the measure applied to subgroups. Bourguignon (1979) showed that the Gini coefficient, together with other proposed metrics, does not have this property.

But a more contextual problem with the Gini index is that it lacks direction as to the source of the inequality. Specifically, it does not properly pick up the kind of inequality that would concern most observers, namely positive distribution skewness, meaning too many people on low incomes. Following the precept of Dalton (1920) that any measurement of inequality has implicitly a social welfare reference, Atkinson (1970) also Foster et al. (1984), address the direction problem by means of an observer chosen parameter that reflects a greater, or lesser, observer preoccupation with the positive skewness in the income distribution. Formally, the Atkinson index can be transformed to the Theil index and thus both indices belong to the class of the generalized entropy measures, which is a single parameter family (Jenkins \& Van Kerm, 2009). In turn, there exist generalizations from the single parameter family to a two parameter family (Foster \& Shneyerov, 1999). However, a quite different tack to the directional problem has been taken in recent contribution by Bowden (2016a). His $v$-index (standing for disadvantage or envy) looks at inequality from the point of view of the subjects themselves; an internal rather than external vantage point. On the average, do people think that others are better off, or worse off, than themselves? The resulting metric can be simply expressed in terms of the means of ancillary distributions, the left and right unit entropic shifts of the original. As a welfare oriented asymmetry metric, the $v$-metric will be employed as a point of departure in the present paper.

A more complete approach of this kind would also have to pay attention to two further welfare aspects. One is the dispersion of the income distribution, as a 'noticeability' property. Wide dispersion attracts more attention to very high or very low incomes, in this context relative to my own. A second is the average income itself. If times are good, higher incomes in others attract less opprobrium. There is a correspondence here with managerial remuneration practices; if the firm is doing well, and stockholder rewards are good, then a hike in executive remuneration is carried, even with acclaim, at the company AGM.

All this suggests that three key metrics are involved in social judgements about income distribution: the mean (or median), the skewness, and the dispersion (spread). All are tacitly embodied in Sen's welfare metric, for the Gini index itself can be shown to depend 
on both the asymmetry and the spread (Bowden, 2016a). The problem is that the Gini index does not separate out the two, or more precisely, the direction of the asymmetry. A cardinal utility analysis would call on some optimum weighting of the three welfare dimensions: mean, asymmetry, and dispersion. But in an ordinal framework, one can adopt an indifference curve approach, to some degree reminiscent of the ordinal utility Bergson-Samuelson framework for a social welfare function. This might not be so useful for direct inter-country comparisons as such. But by longitudinal comparisons over time in the three key metrics, one can compare experiences between countries, as a matter of comparative welfare economics.

Comparisons of this kind are facilitated if the metrics for asymmetry and spread fall within a common framework. The present contribution provides such a basis in terms of metric duality, which in this context means that the two metrics can be obtained by a simple internal change of sign. This ensures both dimensional conformity and a convenient phase plane diagram over time.

The empirical application utilises such a framework to study changes that took place pre- and post- the Global Financial Crisis in Europe. This is not to say that the GFC and its aftermath were by any means the sole causal influence. These were also the times of structural change arising from import competition, the oil price rise and (incipient) fall, and other contributing factors. The result was some dramatic changes in income distribution metrics and implicitly, the rise or fall in their latent social indifference level surfaces (section 2.3). Nor were these changes equally severe across the respective countries. The empirical work of the present paper translates the experiences of 15 of the major European countries in the form of dynamic phase planes between directed asymmetry and spread. It turns out that there are marked differences, which can be categorised into two groups, the risers ('bad') and the fallers ('good'). These tend to be uncompensated by relative difference in their respective mean income series.

The scheme of the paper is as follows. Section 2 is a methodological review, establishing the dual asymmetry and spread metrics, followed by a short discussion of their use as joint proxies for social welfare. Section 3 is the empirical core of the paper. Spread and asymmetry phase planes are graphed for fifteen major European countries between 2005 and 2013. Accompanying tables and discussion highlight similarities and differences in their respective experiences over this interval. Section 4 contains some concluding remarks.

\section{Methodological review}

In what follows the conceptual basis is reviewed, starting with the metrics for disadvantage as directed asymmetry, together with spread. Mathematical details are kept to a minimum; a more comprehensive treatment can be found in Bowden (2012, 2016a,b), also Kraemer (2016) for the axiomatic basis as an income inequality metric. Section 2.3 turns to consideration of the social welfare; the asymmetry and spread metrics for any 
given year can be viewed as analogous to points on a social welfare indifference curve. Over successive years this curve may itself change, with social welfare shifts as an outcome.

\subsection{The asymmetry metric}

Let $y$ be the income random variable, defined on $\mathbb{R}_{+}$with distribution function $F(y)$ and density $f(y)$. Suppose my own income is $y$. The metric for asymmetry derives from comparisons between incomes above mine and those below. The average of those below is the lower conditional expectation $\mu_{l}(y)=\mathbb{E}[Y \mid Y \leq y]$ and for those above, $\mu_{r}(y)=\mathbb{E}[Y \mid Y>y]$. Relative to those above and below, my net disadvantage is

$$
v(y)=\left(\mu_{r}(y)-y\right)-\left(y-\mu_{l}(y)\right)
$$

Taking the expected value over the entire distribution $F(y)$ gives a scalar metric that represents the average degree of peer relative advantage:

$$
v=\mathbb{E}(v(y))=\mu_{L}+\mu_{R}-2 \mu .
$$

Here $\mu$ is the mean of the original or natural income distribution. The two terms $\mu_{L}, \mu_{R}$ also refer to means, but in this case of ancillary distributions with densities defined by

$$
f_{L}(y)=\xi_{L}(y) f(y) ; \quad f_{R}(y)=\xi_{R}(y) f(y),
$$

where $\xi_{L}(y)=-\ln F(y) ; \quad \xi_{R}(y)=-\ln (1-F(y))$ are factors (technically, RadonNikodym derivatives) that shift the original distribution respectively to the left and right. The corresponding distribution functions are given by

$$
F_{L}(y)=F(y)\left(1+\xi_{L}(y)\right) ; \quad 1-F_{R}(y)=(1-F(y))\left(1+\xi_{L}(y)\right)
$$

In such terms, the metric 2 becomes easy to compute, starting with the original histogram for the income distribution. Expression 2 can be more compactly expressed as $v=2\left(\mu_{c}-\mu\right)$, where $\mu_{c}$ is the mean of the centred or average shift $F_{c}(y)=$ $\left(F_{L}(y)+F_{R}(y)\right) / 2$. Noting that $\mu, \mu_{l}, \mu_{r}$ are all commensurate first moments, the standardisation $\tilde{v}=v / \mu$ is recommended for time series or cross section applications.

The resulting metric $v$ is explicitly a directional asymmetry metric. A value $v>0$ means that the distribution is positively skewed, so that $\mu>x_{m}$, the median, while if $v<0$ the reverse is true: $\mu<x_{m}$. Unlike textbook skewness diagnostics such as the third order moment, the $v$-metric has contextual reference, in this case to how people feel about their comparative income. Additionally, since the existence of higher moments in income distributions cannot be granted in general (Kleiber, 1997), it is possible to assess skewness for a more extended set of distributions.

Figure 1 illustrates with the Norwegian monthly disposable income data (truncated 
at 200,000 NOK). Over the time span 2005 - 2013 the distributions have become more symmetric, manifested by the corresponding $\mathrm{v}$ metrics, though still with some way to go towards perfect symmetry $(v=0)$. It should further be noted that the mean has increased over the years. The standardised values $\tilde{v}$ are respectively $0.72,0.17$ and 0.11 (cf. table 3).

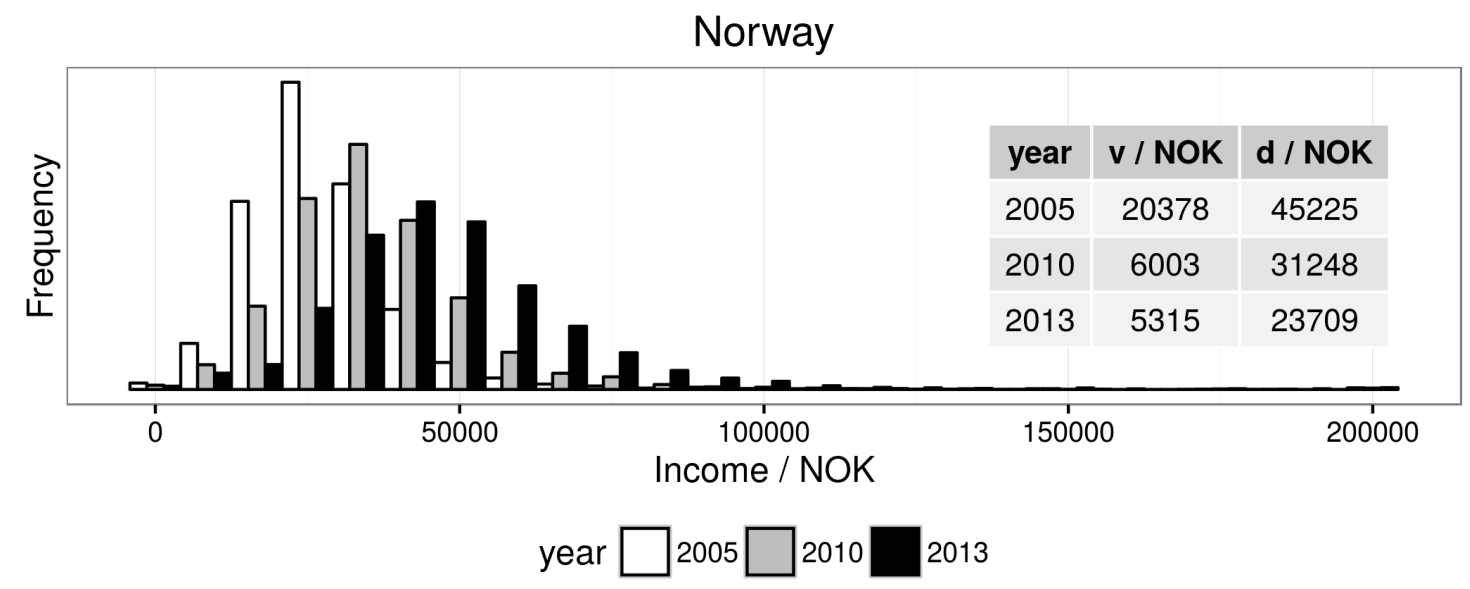

FIG. 1: Norway: monthly disposable income (1 NOK 0.12 USD).

\subsection{The dual dispersion metric}

A metric for dispersion or distribution spread that is dual with respect to the $v$-metric is derived by simply changing the separating sign in expression 1 . Thus the function

$$
\left(\mu_{r}(y)-y\right)+\left(y-\mu_{l}(y)\right)
$$

can be interpreted as a dispersion penalty function. This is a two sided function rising away from the median in either direction. Its expected value is given by

$$
d=(E)(d(y))=\mu_{R}-\mu_{L}
$$

Note that $d>0$ and by construction, $d>v$. In turn, this can be standardised as $\tilde{d}=$ $d / \mu$. Several rationales exist for the interpretation of $d$ as a spread or dispersion metric. It is equal to the total area under the partition entropy function, which refers to the distribution of uncertainty as to whether $Y \leq y$ or $Y>y$ as $y$ varies along its range. Bowden $(2012,2016 b)$ contains further details as to this link. A related rationale runs in terms of the accumulation function of stochastic dominance theory. A distribution for which $F(y)$ slopes more gradually will always have a higher value for the metric $d$.

For the Norwegian example as in figure 1, the $d$ measure has decreased over the given horizon. The effect is accentuated in the standardised versions $\tilde{d}$, where the values are $1.47,0.86$ and 0.79 (cf. table 3 ). So the net effect, taken in conjunction with $\tilde{d}$, is for a narrowing of the distribution spread together with diminishing asymmetry. 


\subsection{Level surfaces}

The three metrics, namely $\mathrm{v}$ for asymmetry and $\mathrm{d}$ for spread, together with $\mu$ (or the median) for central tendency, provide cues for thinking how the average person might react to the publication of income data or an income distribution histogram. A positive skewness indicator $\mathrm{v}$ will indicate that the average person is net disadvantaged relative to those below and above him or her on the scale. This will be more noticeable where the distribution has a wider spread. And in both cases the reaction will be moderated when the average worker's income has itself risen.

The foregoing suggests an ordinal social welfare function, geared to the average worker, of the general form

$$
S W=\Psi(\mu, v, d),
$$

with $\varphi_{1}=\frac{\partial \Psi}{\partial \mu}>0 ; \quad \varphi_{2}=\frac{\partial \Psi}{\partial v} \lessgtr 0 ; \quad \varphi_{3}=\frac{\partial \Psi}{\partial d}<0$. As a further aid to interpretation, suppose that $\varphi$ is separable between $\mu$ and $v, d$ so:

$$
S W=\Psi(\mu, \varphi(\tilde{v}, \tilde{d})) .
$$

This would certainly be the case if the parent function 7 was homogenous in its three arguments, but it is not necessary to introduce such a restriction.

As it stands, the form 8 could be interpreted as saying that the average worker looks first at the spread and asymmetry in relation to his or her own income, then modifies any reaction if the personal income is higher or lower during any given year. For any given income there is therefore a set of indifference curves (level surfaces) as between spread and skewness. However, these may not be uniformly concave or convex. For as skewness $(v)$ becomes more positive, it requires progressively lower dispersion $(d)$ to materially lessen the envy. By way of contrast, if $v$ becomes more negative, it might require a progressively higher spread to preserve the same social utility. So the social indifference curves relating skewness to spread might therefore be sigmoid in shape.

\section{The European experience: temporal phase plane}

During the years 2005 - 2013 Europe passed through a cycle of boom and in some cases, bust. Although there was a common exposure to the GFC, recovery was far from universal across the zone. In part this was due to differing exposures to private and public sector debt. In addition, specific casual influences differed as between individual countries. Oil prices recovered quickly between 2009 and 2013, to the benefit of producers such as Norway, the Netherlands and the UK. A number of countries, including Poland and the Czech Republic joined the EU in the course of its 2004 enlargement. Some countries were impacted more than others by the rise of manufactured imports from China and Vietnam. Data availability precludes coverage of subsequent events such as 
the collapse of oil prices and more recently the refugee crisis.

The empirical analysis that follows is based on data drawn from Eurostat, EU Statistics on Income and Living Conditions (2013). We use the monthly disposable income on household level in local currencies, with overall an average of 6,875 observations per year and country. The subset selected for detailed analysis covers 15 European countries between 2004 and 2013.

\subsection{Dynamics with the Gini index}

Following the discussion of section 1, a preliminary check is whether the Lorenz curves do intersect in our dataset. Table 1 shows the number of intersections for the Lorenz curves in the case of Germany. In only five out of 28 combinations do the curves not intersect. Since the assumptions about the relative variance required for intersecting curves are not met, it would therefore not be legitimate to compare the Gini coefficients between intersecting years. Furthermore when moving to a cross-sectional perspective, the number of comparisons of Lorenz curves will increase exponentially and with it the incommensurability of the Gini's within time or across countries.

ТАв. 1: Intersections of Lorenz curves for the disposable income in Germany ${ }^{\alpha}$

\begin{tabular}{rrrrrrrrr}
\hline 2006 & 2007 & 2008 & 2009 & 2010 & 2011 & 2012 & 2013 & \\
\hline 1 & 1 & 1 & 1 & 5 & 1 & 4 & 5 & 2005 \\
& 5 & 3 & 3 & 2 & 2 & 2 & 3 & 2006 \\
& & 5 & $0^{*}$ & 2 & 2 & $0^{*}$ & 1 & 2007 \\
& & & 2 & $0^{*}$ & 4 & $0^{*}$ & 2 & 2008 \\
& & & & 2 & 10 & 2 & 3 & 2009 \\
& & & & & 1 & 2 & 5 & 2010 \\
& & & & & & $0^{*}$ & 3 & 2011 \\
& & & & & & & 3 & 2012 \\
\hline
\end{tabular}

${ }^{\alpha}$ Shown are the number of intersections points when comparing the Lorenz curves calculated from the income distribution of Germany for the corresponding years.

*Combinations without intersection Lorenz curves.

\subsection{Dynamics in the $v$-d plane}

A dynamic phase plane analysis turns out to be an effective way of highlighting both the similarities and differences. For space reasons the phase diagrams are reproduced only for the more major economics, or else those that were subject to particular stress. Among the latter, Greece could not be included because of very limited data availability. Diagrams for all the remaining European countries can be obtained from the authors.

Formats in figure 1 plot the normalised $v$ metrics against those for spread on the horizontal axis. No country has a negative $v$ metric, hence the positive vertical half axis. 
The countries differ as to the observed range of the normalised asymmetry and spread metrics $\tilde{v}, \tilde{d}$, and this is reflected in the axis ranges of the respective diagrams. As a visual aid, directional links are employed to make the time sequence more identifiable. The respective time periods of data availability can be identified from the beginning and end dates of these sequences.

\subsection{Discussion}

As a useful if somewhat imprecise generalisation, the respective $v$ - $d$ dynamics can be categorised into three groups, provisionally labelled the 'inequality risers', the 'inequality fallers'; and 'mixed' where the trend is less clear. Table 2 lists the countries in this format. Further details about these groups and their constituents are provided in Table 3. There, the first column gives the average GDP per capita change over the available data horizon for each country as the third dimension of welfare analysis (cf. expression 8), together with the overall ranking of the growth. There is no clear association on this count between inequality risers and fallers. Also reported are the asymmetry and dispersion parameters before, at, and after the GFC together with the corresponding ranking within the 15 countries. Comparative rankings are not automatically responsive to individual metric changes, though there is some effect with inequality increases.

What the data do show is that the impact of economic events has varied between the European countries. Bad economic times in countries such as Italy, Spain, and France have been affected more at the low end of the income scale; while in Portugal the burden has been spread more uniformly. This is also true of Germany and the Netherlands, even though the economic impact was more muted. Newer members of the European Union have fared better in this respect. The Czech Republic, Hungary, and Poland, all admitted in the 2004 Enlargement, share a common experience of falling income inequality and a healthy average GDP growth rate.

ТАв. 2: Secular inequality trends over the sample period from $2004-2013$

\begin{tabular}{ccc}
\hline Inequality risers & Inequality fallers & Mixed \\
$\mathrm{v}, \mathrm{d}$ rise over time & $\mathrm{v}, \mathrm{d}$ fall over time & \\
\hline France & Czech Republic & Austria \\
Italy & Hungary & Belgium \\
Spain & Ireland & Germany \\
Sweden & Norway & Netherlands \\
& Poland & \\
& Portugal & \\
& United Kingdom \\
\hline
\end{tabular}




\section{Concluding remarks}

The asymmetry - spread phase plane diagrams of figure 1 reveal significant differences over the data horizon. It is easy enough to rationalise the experience of some countries, Norway being an obvious example with the strength of oil prices over the interval. Likewise, joining the EU has on the face of it been good, with fallers such as the Czech Republic or Hungary reflecting rising employment and wages as tariffs are dismantled and trade opened up.

But other comparisons are more problematical, even those that one might expect to be similar. Why is France a riser, which one might consider bad, but neighbouring Germany is not, but corresponds to the mixed group? Likewise both Spain and Portugal suffered from the GFC, but why is Spain a $v-d$ riser while the Portugal is a faller? Macroeconomic data, such as unemployment, or the public and private debt to GDP ratio, do not appear to throw much light on such issues. Structural impediments to the adjustment of wages or employment might have been of more importance in countries such as France, with its public sector rigidities.

To summarise, the metrics and phase diagrams do not in themselves resolve issues of this kind. But in throwing up differences in such a dramatic way, they do motivate the search for answers on a more structural level. Over time there will arise fresh circumstantial challenges, whether these arise from global fallouts, localised trading blocs, or political events on a national level. If nothing else, the message of the present paper is that one way or another, these do have an observable welfare impact on the economic shares of society. 


\section{A. Figures and Tables}
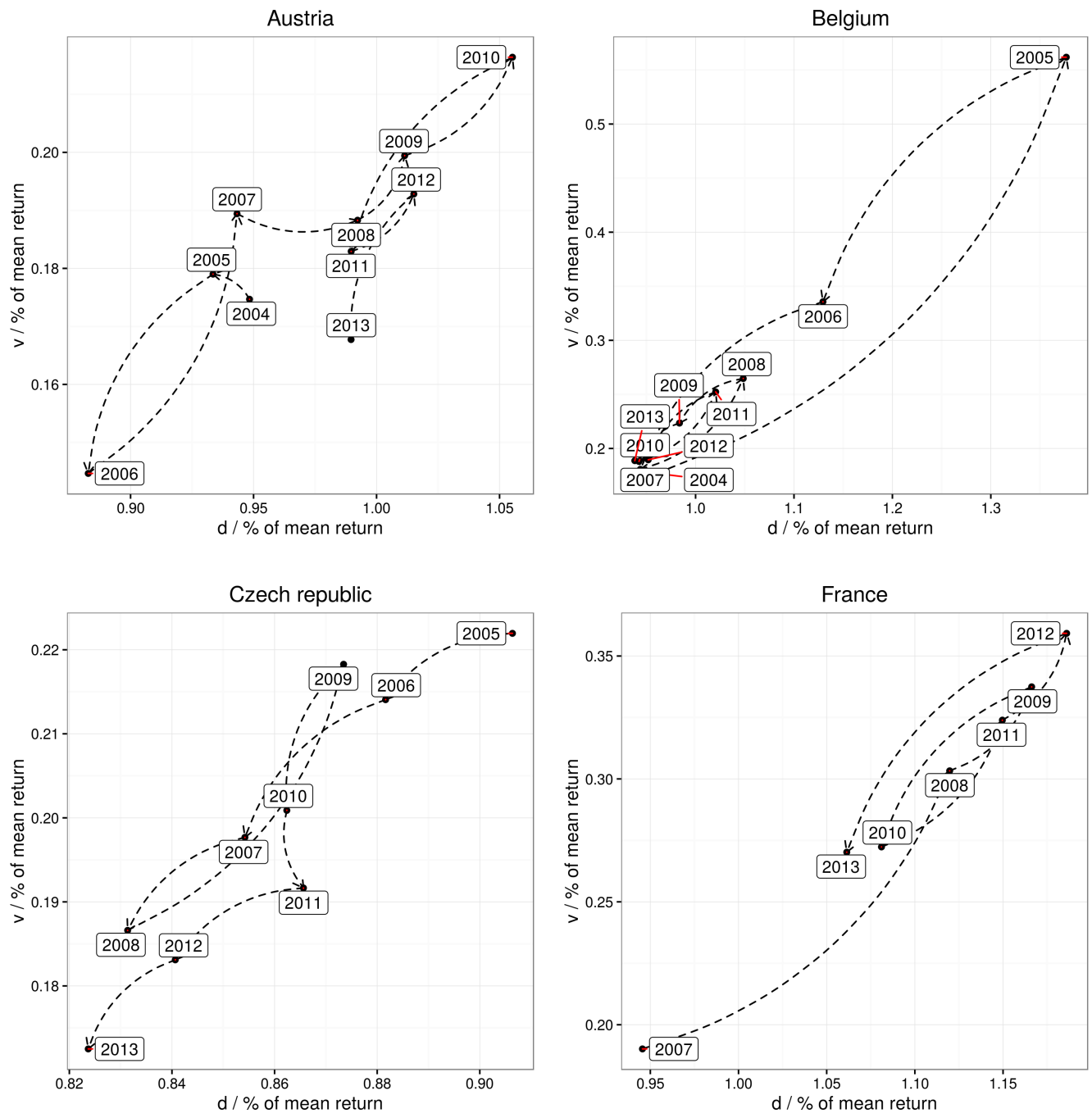

FIG. 2: Phase diagrams for the different countries for the complete time interval. 

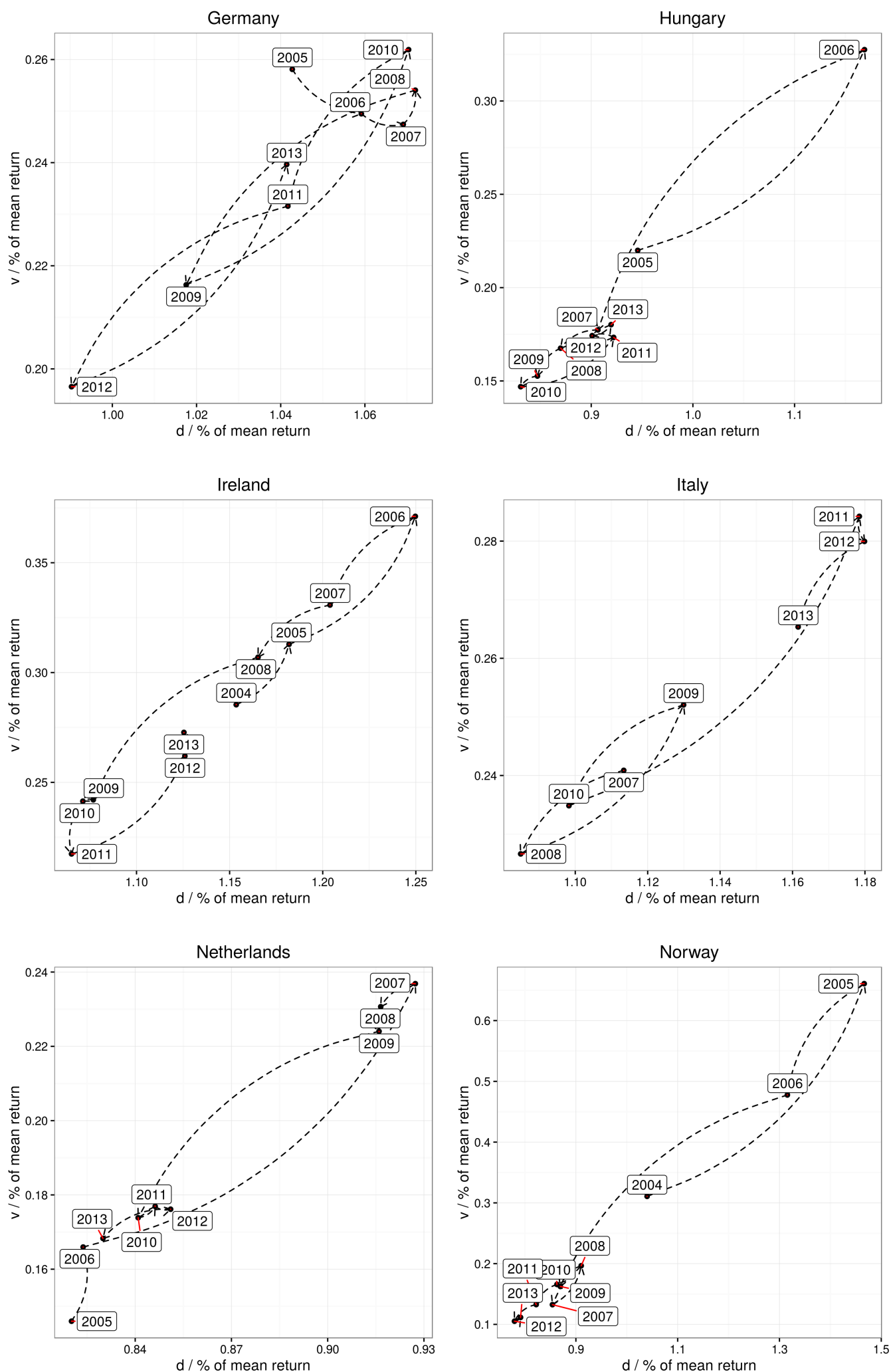

FIG. 1: cont'd: Phase diagrams 

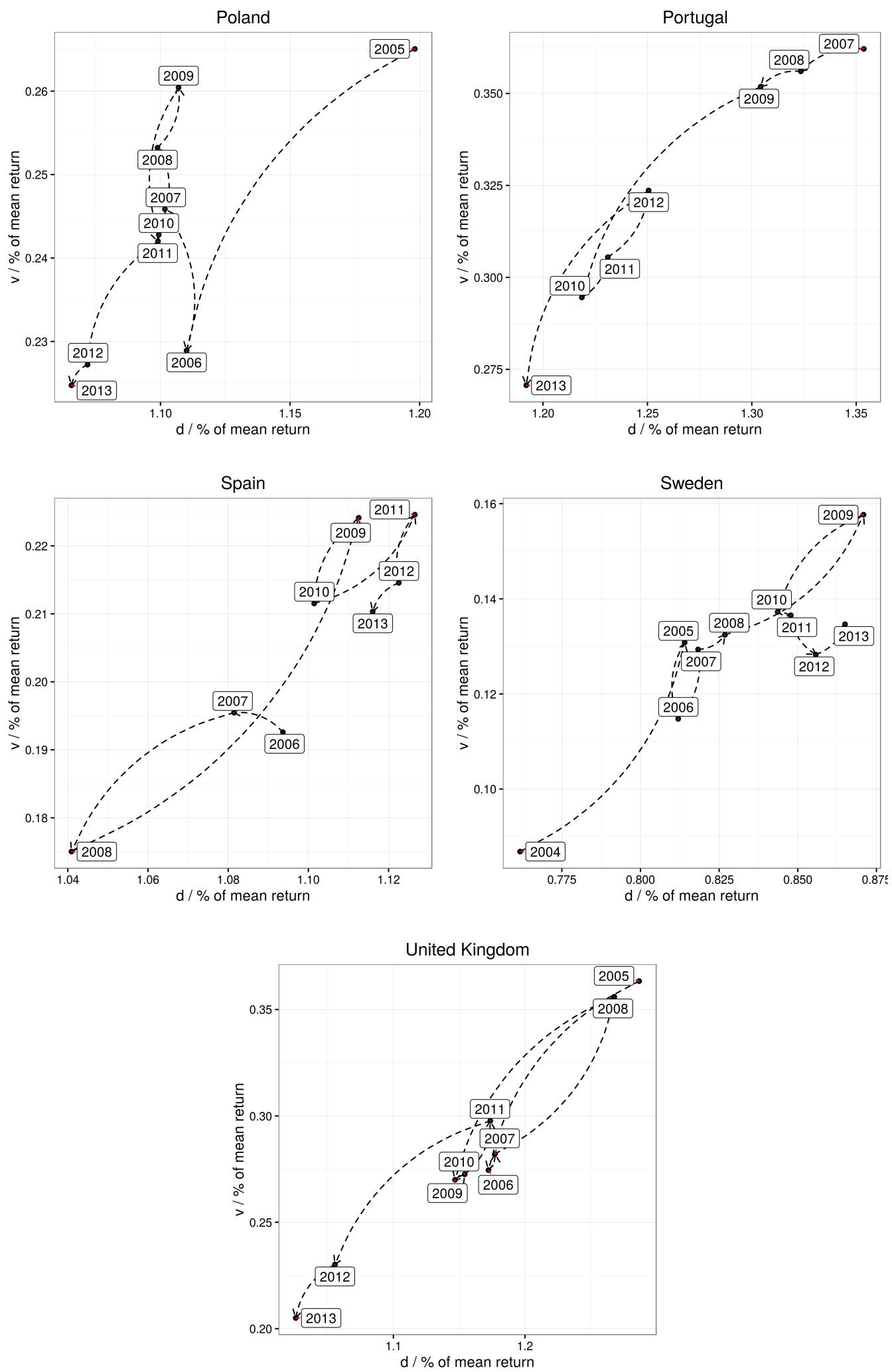

FIG. 0: cont'd: Phase diagrams 
ТАв. 3: Results of the proposed new inequality measure with ranking ${ }^{\alpha}$

\begin{tabular}{|c|c|c|c|c|c|c|c|c|c|c|c|c|c|c|}
\hline & $\begin{array}{c}\text { Av. Change GDP / } \\
\text { \% p.cap }\end{array}$ & Rank & 2005 & $\begin{array}{l}d / \mu \\
2010\end{array}$ & 2013 & 2005 & $\begin{array}{l}\text { Rank } \\
2010\end{array}$ & 2013 & 2005 & $\begin{array}{l}d / \mu \\
2010\end{array}$ & 2013 & 2005 & $\begin{array}{l}\text { Rank } \\
2010\end{array}$ & 2013 \\
\hline \multicolumn{15}{|c|}{ Risers } \\
\hline $\mathrm{FR}^{*}$ & 1.06 & 12 & 0.95 & 1.08 & 1.06 & 5 & 10 & 10 & 0.2 & 0.3 & 0.3 & 4 & 13 & 13 \\
\hline $\mathrm{IT}^{*}$ & -0.28 & 15 & 1.11 & 1.1 & 1.16 & 9 & 11 & 14 & 0.2 & 0.2 & 0.3 & 8 & 9 & 12 \\
\hline ES* & 0.77 & 13 & 1.08 & 1.1 & 1.12 & 8 & 13 & 12 & 0.2 & 0.2 & 0.2 & 5 & 7 & 9 \\
\hline SE & 1.94 & 4 & 0.81 & 0.84 & 0.87 & 1 & 3 & 4 & 0.1 & 0.1 & 0.1 & 1 & 1 & 2 \\
\hline \multicolumn{15}{|c|}{ Fallers } \\
\hline$C Z$ & 2.41 & 2 & 0.91 & 0.86 & 0.82 & 3 & 4 & 2 & 0.2 & 0.2 & 0.2 & 7 & 6 & 5 \\
\hline HU & 1.28 & 10 & 0.95 & 0.83 & 0.92 & 6 & 1 & 5 & 0.2 & 0.1 & 0.2 & 6 & 2 & 6 \\
\hline IE & 2.22 & 3 & 1.18 & 1.07 & 1.13 & 10 & 9 & 13 & 0.3 & 0.2 & 0.3 & 11 & 10 & 15 \\
\hline NO & 1.65 & 5 & 1.47 & 0.86 & 0.79 & 15 & 5 & 1 & 0.7 & 0.2 & 0.1 & 15 & 3 & 1 \\
\hline $\mathrm{PO}$ & 3.95 & 1 & 1.2 & 1.1 & 1.07 & 11 & 12 & 11 & 0.3 & 0.2 & 0.2 & 10 & 11 & 10 \\
\hline $\mathrm{PT}^{*}$ & -0.02 & 14 & 1.35 & 1.22 & 1.19 & 13 & 15 & 15 & 0.4 & 0.3 & 0.3 & 12 & 15 & 14 \\
\hline UK & 1.45 & 6 & 1.29 & 1.15 & 1.03 & 12 & 14 & 8 & 0.4 & 0.3 & 0.2 & 13 & 14 & 8 \\
\hline \multicolumn{15}{|c|}{ Mixed } \\
\hline $\mathrm{AT}$ & 1.43 & 8 & 0.93 & 1.06 & 0.99 & 4 & 7 & 7 & 0.2 & 0.2 & 0.2 & 3 & 8 & 3 \\
\hline $\mathrm{BE}$ & 1.45 & 6 & 1.38 & 0.94 & 0.94 & 14 & 6 & 6 & 0.6 & 0.2 & 0.2 & 14 & 5 & 7 \\
\hline $\mathrm{DE}$ & 1.31 & 9 & 1.04 & 1.07 & 1.04 & 7 & 8 & 9 & 0.3 & 0.3 & 0.2 & 9 & 12 & 11 \\
\hline NL & 1.07 & 11 & 0.82 & 0.84 & 0.83 & 2 & 2 & 3 & 0.1 & 0.2 & 0.2 & 2 & 4 & 4 \\
\hline
\end{tabular}

${ }^{\alpha}$ The table shows the average GDP growth from 2005 to 2013 , the scaled dispersion and skewness measures before the GFC (taken as 2005*), just after the GFC (taken as 2010) and most recent available (2013).

*2005 data not available for France, Italy, Portugal. Provided data are from 2007. 


\section{Literature}

Atkinson, A. B. (1970). On the measurement of inequality. Journal of Economic Theory, 2, 244-263.

Bergson, A. (1938). A Reformulation of Certain Aspects of Welfare Economics. The Quarterly Journal of Economics, 52(2), 310-334.

Bourguignon, F. (1979). Decomposable income inequality measures. Econometrica: Journal of the Econometric Society, 47, 901-920.

Bowden, R. J. (2012). Information, measure shifts and distribution metrics. Statistics, 46(2), 249-262.

Bowden, R. J. (2016a). Dual spread and asymmetry distribution metrics based in partition entropy. Kiwcap Research Ltd, (pp. 1-13).

Bowden, R. J. (2016b). Giving Gini direction: An asymmetry metric for economic disadvantage. Economics Letters, 138, 96-99.

Conceição, P. \& Ferreira, P. (2000). The young person's guide to the Theil index: Suggesting intuitive interpretations and exploring analytical applications. UTIP Working Paper Number 14, (pp. 1-54).

Dalton, H. (1920). The Measurement of the Inequality of Incomes. The Economic Journal, 30(119), 348-361.

Davies, J. \& Hoy, M. (1995). Making Inequality Comparisons When Lorenz Curves Intersect. The American Economic Review, (pp. 980-986).

Donaldson, D. \& Weymark, J. A. (1980). A single-parameter generalization of the Gini indices of inequality. Journal of Economic Theory, 22(1), 67-86.

Foster, J., Greer, J., \& Thorbecke, E. (1984). A Class of Decomposable Poverty Measures. Econometrica: Journal of the Econometric Society, (pp. 761-766).

Foster, J. E. (1983). An axiomatic characterization of the Theil measure of income inequality. Journal of Economic Theory, 31(1), 105-121.

Foster, J. E. \& Shneyerov, A. A. (1999). A general class of additively decomposable inequality measures. Economic Theory, 14(1), 89-111.

Goodman, L. a. \& Arrow, K. J. (1953). Social Choice and Individual Values. American Sociological Review, 18(1), 116.

Greselin, F. \& Zitikis, R. (2015). Measuring economic inequality and risk: a unifying approach based on personal gambles, societal preferences and references. arXiv:1508.00127. 
Jenkins, S. P. \& Van Kerm, P. (2009). The measurement of economic inequality. Oxford University Press.

Kleiber, C. (1997). The existence of population inequality measures. Economics Letters, 57(1), 39-44.

Kraemer, W. (1998). Measurement of inequality. Handbook of Applied Economic Statistics, (pp. 39$)$.

Kraemer, W. (2016). Beyond inequality: A novel measure of skewness and its properties. Working Paper, TU Dortmund University.

Samuelson, P. A. (1947). Foundations of Economic Analysis. Harvard University Press.

Sen, A. (1970). Collective Choice and Social Welfare. San Francisco: Holden Day.

Theil, H. (1965). The Information Approach to Demand Analysis. Econometrica: Journal of the Econometric Society, 33(1), 67-87.

Yitzhaki, S. (1983). On an Extension of the Gini Inequality Index. International Economic Review, 24(3), 617-628. 

